\title{
Overexpression of DAZL, STRA8, and BOULE Genes and Treatment With BMP4 or Retinoic Acid Modulate the Expression of MSC Overexpressing Germ Cell Genes
}

\author{
Paloma Cordero ${ }^{1}$, Alejandra Guerrero-Moncayo ${ }^{1}$, Monica De los Reyes ${ }^{1}$, \\ Manuel Varas-Godoy ${ }^{2}$, Jahaira Cortez ${ }^{1}$, Cristian G. Torres ${ }^{3}$, Victor H. Parraguez ${ }^{4}$ and \\ Oscar A. Peralta ${ }^{1 *}$
}

1 Department of Animal Production Sciences, Faculty of Veterinary and Animal Sciences, University of Chile, Santiago, Chile, ${ }^{2}$ Centro de Biología Celular y Biomedicina (CEBICEM), Facultad de Medicina y Ciencia, Universidad San Sebastián, Santiago, Chile, ${ }^{3}$ Department of Clinical Sciences, Faculty of Veterinary and Animal Sciences, University of Chile, Santiago, Chile, ${ }^{4}$ Department of Biological Sciences, Veterinary and Animal Sciences, University of Chile, Santiago, Chile

\section{OPEN ACCESS}

Edited by:

Slavko Mojsilovic,

University of Belgrade, Serbia

Reviewed by:

Carlos Eduardo Ambrósio,

University of São Paulo, Brazil

Takashi Taguchi,

Western University of Health

Sciences, United States

*Correspondence:

Oscar A. Peralta

operalta@uchile.cl

Specialty section:

This article was submitted to Veterinary Regenerative Medicine,

a section of the journal

Frontiers in Veterinary Science

Received: 13 February 2021 Accepted: 12 April 2021

Published: 25 May 2021

Citation:

Cordero P, Guerrero-Moncayo A, De

Ios Reyes M, Varas-Godoy M,

Cortez J, Torres CG, Parraguez VH and Peralta OA (2021) Overexpression of DAZL, STRA8, and BOULE Genes and Treatment With BMP4 or Retinoic Acid Modulate the Expression of MSC Overexpressing Germ Cell Genes.

Front. Vet. Sci. 8:667547.

doi: 10.3389/fvets.2021.667547
In vitro gamete derivation from stem cells has potential applications in animal reproduction as an alternative method for the dissemination of elite animal genetics, production of transgenic animals, and conservation of endangered species. Mesenchymal stem cells (MSCs) may be suitable candidates for in vitro gamete derivation considering their differentiative capacity and their potential for cell therapy. Due to its relevance in gametogenesis, it has been reported that retinoic acid (RA) and bone morphogenetic protein (BMP) 4 are able to upregulate the expression of specific markers associated to the early stages of germ cell (GCs) differentiation in bovine fetal MSCs (bfMSCs). In the present study, we used polycistronic vectors containing combinations of GC genes DAZL, STRA8, and BOULE followed by exposure to BMP4 or RA to induce GC differentiation of bovine fetal adipose tissue-derived MSC (AT-MSCs). Cells samples at Day 14 were analyzed according to the expression of pluripotent genes NANOG and OCT4 and GC genes DAZL, STRA8, BOULE, PIWI, C-KIT, and FRAGILIS using Q-PCR. Fetal and adult testis and AT-MSCs samples were also analyzed for the expression of DAZL, STRA8, and NANOG using immunofluorescence. Increased gene expression levels in the adult testis and cell-specific distribution of DAZL, STRA8, and NANOG in the fetal testis suggest that these markers are important components of the regulatory network that control the in vivo differentiation of bovine GCs. Overexpression of DAZL and STRA8 in bi-cistronic and DAZL, STRA8, and BOULE in tri-cistronic vectors resulted in the upregulation of OCT4, NANOG, and PIWIL2 in bovine fetal AT-MSCs. While BMP4 repressed NANOG expression, this treatment increased DAZL and c-KIT and activated FRAGILIS expression in bovine fetal AT-MSCs. Treatment with RA for 14 days increased the expression of DAZL and FRAGILIS and maintained the mRNA levels of STRA8 in bovine fetal AT-MSCs transfected with bi-cistronic and tri-cistronic vectors. Moreover, RA treatment repressed the expression of OCT4 and NANOG in these cells. Thus, overexpression of DAZL, STRA8, and BOULE induced the upregulation of the pluripotent 
markers and PIWIL2 in transfected bovine fetal AT-MSCs. The partial activation of GC gene expression by BMP4 and RA suggests that both factors possess common targets but induce different gene expression effects during GC differentiation in overexpressing bovine fetal AT-MSCs.

Keywords: mesenchymal stem cells, bovine fetus, germ cell differentiation, polycistronic vector, bone morphogenetic protein 4, retinoic acid

\section{INTRODUCTION}

In vitro gamete derivation from stem cells has a promising potential role for the treatment of male infertility in human reproductive medicine $(1,2)$. This technology may also expand its potential applications to animal reproduction as an alternative method for the dissemination of elite animal genetics, production of transgenic animals, and conservation of endangered species. In the case of a cattle, large-scale culture of in vitro-produced germ cells (GCs) would allow a continuous supply of elite animal genetics for transplantation into the seminiferous tubules of recipient animals in a donor-derived spermatogenesis (3).

Mesenchymal stem cells isolated from bovine fetal tissues (bfMSCs) posses the intrinsic capacity for differentiation into osteogenic, chondrogenic, and adipogenic lineages $(4,5)$. Plasticity of bfMSCs is not limited to mesodermal derivatives since these cells were also able to differentiate into neuroectodermal and endodermal cell types (6). Recently, it has been reported that bfMSCs exposed to bioactive factors, including retinoic acid (RA) and bone morphogenetic protein (BMP) 4 or cocultured with Sertoli cells, are able to upregulate the expression of specific markers associated to the early stages of GC differentiation $(7,8)$. In addition, bfMSCs can be easily isolated and expanded under in vitro conditions to obtain a sufficient number for transplantation. Moreover, bfMSCs express low levels of immunogenic markers (MHC-I, MHC-II, CD80, and CD86), display immune suppressive capacity (9), and possess a reduced intrinsic teratogenic potential (10). Overall, these features suggest that bfMSCs may be used for in vitro GC production and allogenic transplantation into the testis of recipient animals.

In domestic animals, male GCs are derived from a population of primordial germ cells (PGCs) which originated in the proximal epiblast (11). PGC specification is induced by extrinsic factors secreted by extraembryonic ectoderm, including BMP4 that activates the expression of GC-specific marker FRAGILIS in the proximal epiblast, marking the first step in germ-line commitment (12). In mice, the subset of presumptive GCs positive for FRAGILIS migrates to the extraembryonic mesoderm and suppresses somatic cell gene expression, promoting the expression of pluripotent genes octamer-binding transforming factor 4 (OCT4) and NANOG (13). During migration through the hindgut to the genital ridge developing into the future testis, PGCs start expressing deleted in azoospermia like (DAZL), which plays essential roles in the development of PGCs and in the differentiation and maturation of GCs (14). After migration, mice PGCs enter a mitotic arrest and are reactivated after birth to initiate spermatogenesis. It has been reported that stimulated by RA8 (STRA8) is an essential factor involved in the decision of meiotic entry of postmigratory PGCs, in a process highly regulated by RA $(15,16)$. Thus, upregulation of STRA8 suggests that the GCs are ready for meiotic pairing and recombination of homologous chromosomes (17).

The relevance of DAZL has been demonstrated in knockout mice, where embryos display a reduced expression of GCspecific genes and postnatal males present an impairment in the progression from $\mathrm{A}$ to A1 spermatogonia and meiotic arrest resulting in azoospermia and sterility $(18,19)$. DAZL is expressed throughout most of the life of GCs and is required for the development of PGCs and for the differentiation and maturation of GCs (14). In addition, BOULE (also called BOLL), a member via RNA-binding protein of the deleted in azoospermia (DAZ) gene family, complements the functions of DAZL and has been reported to be important for meiotic division and spermatogenesis (20). All evidence indicates that DAZL and BOULE have synergistic effects and are fundamental for spermatogenesis (21). Recent studies indicate that the overexpression of STRA8, BOULE, and DAZL promotes the transdifferentiation of goat BM-MSCs to early GC-like cells in vitro $(22,23)$; however, this approach has not been attempted in bfMSCs or bovine adult MSCs. Thus, considering that the co-expression of multiple genes is an interesting strategy for promoting cell differentiation, and the crucial effects of BMP4 and RA on GC development, in the present study, we used polycistronic vectors containing combinations of DAZL, STRA8, and BOULE genes followed by exposure to BMP4 or RA to induce GC differentiation of bovine fetal adipose tissue-derived MSC (AT-MSCs).

\section{MATERIALS AND METHODS}

\section{Study Design}

All procedures have been approved by the Bioethical Committee of the National Commission for Scientific and Technology Research from Chile (Fondecyt). The developmental gene expression of pluripotent OCT4 and NANOG and GC markers DAZL, STRA8, BOULE, PIWI, c-KIT, and FRAGILIS was evaluated in a bovine testis at gestational months 6 (M6) and 9 (M9) and at 1 year (1Y) old. Testis samples were collected from bovine fetuses ( $n=5$ for each stage) and adults $(n=$ 5), and protein expression of DAZL, STRA8, and NANOG were analyzed using immunofluorescence. Furthermore, the effects of the ectopic expression of combinations of DAZL, STRA8, and BOULE using bi-cistronic iDS or tri-cistronic iDSB vectors were evaluated on the potential GC differentiation of bovine fetal AT-MSCs (Figure 1A). Control treatments were 
A

Isolation of AT-MSCs from abattoir-derived bovine fetuses

(7-8 months of gestation)
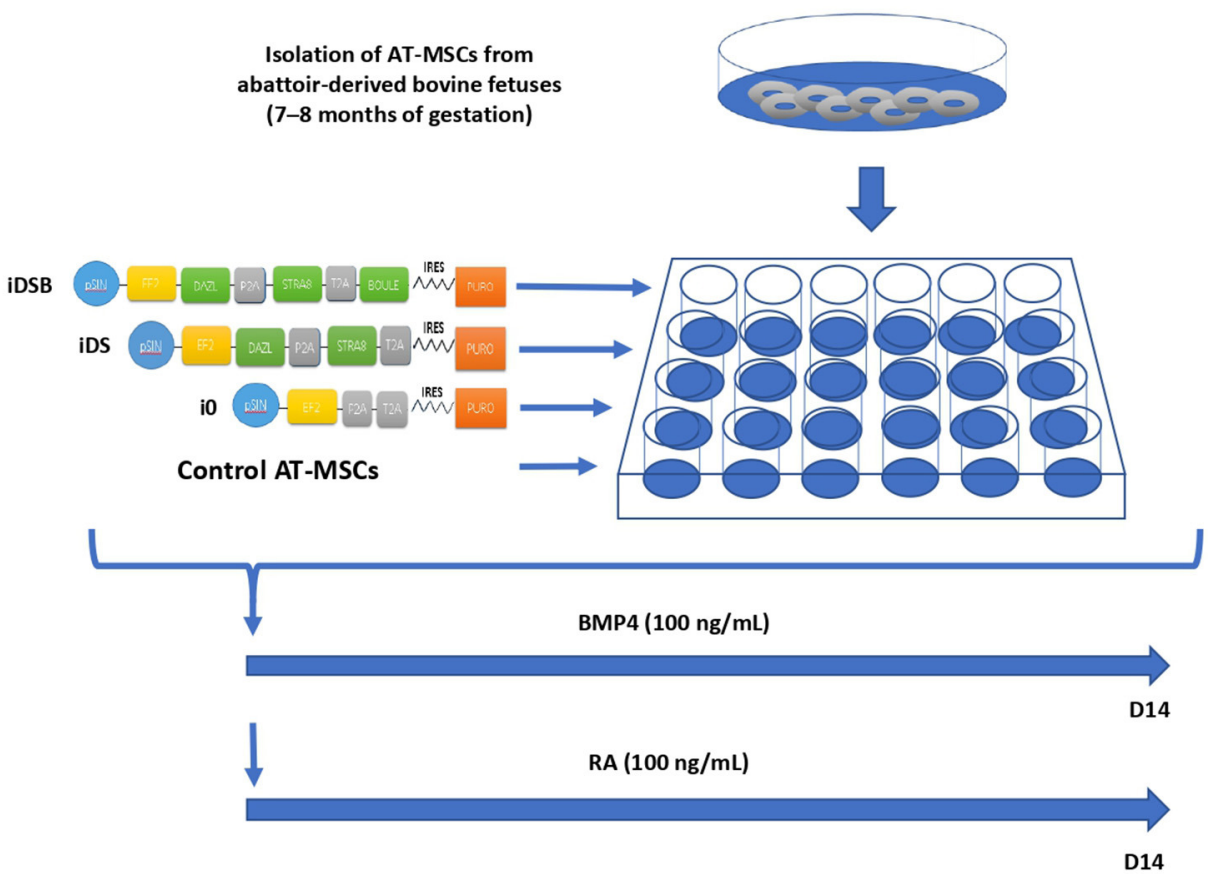

CONTROL
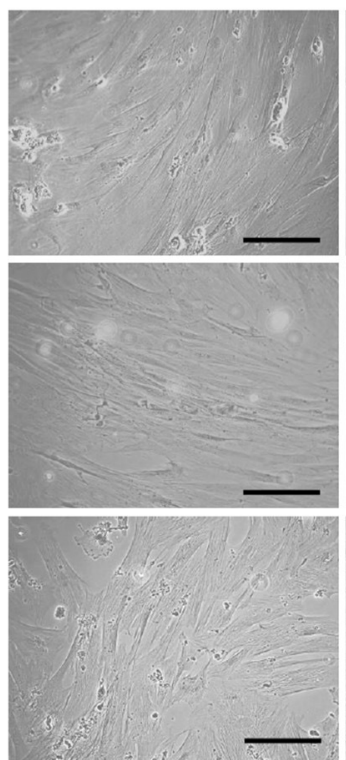
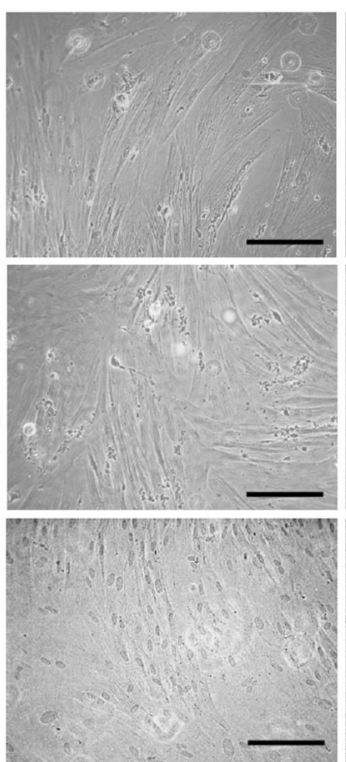

iDS
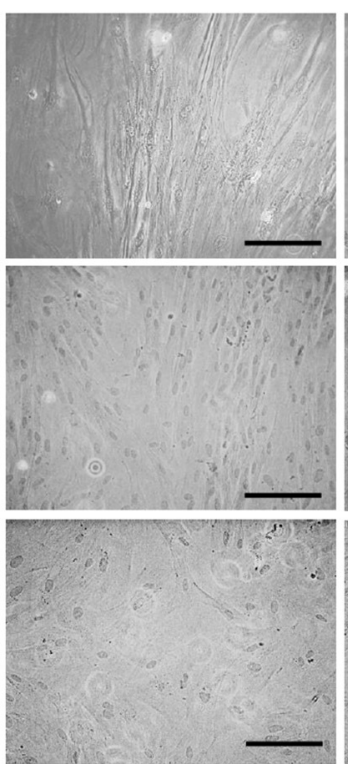

iDSB
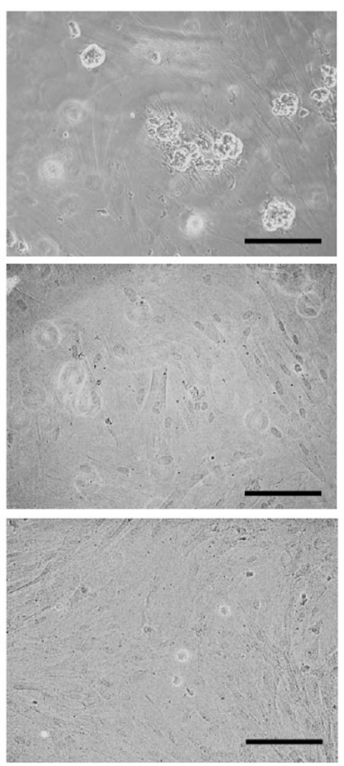

FIGURE 1 | Experimental design for transfection of polycistronic vectors expressing DAZL, STRA8, and BOULE in bovine fetal AT-MSCs and subsequent treatments with BMP4 and RA. (A) AT-MSCs were transfected with tri-cistronic vectors containing a combination of DAZL, STRA8, and BOULE genes (iDSB), bi-cistronic vector carrying DAZL and STRA8 genes (iDS), or empty vector (iO). Control treatment were non-transfected AT-MSCs. Thereafter, transfected and control AT-MSCs were treated with $100 \mathrm{ng} / \mathrm{mL}$ of BMP4 or $0.1 \mu \mathrm{M}$ of RA for 14 days. (B) AT-MSCs expressing i0, iDS, and iDSB had a similar fibroblast-like morphology and culture organization compared to control AT-MSCs. Control AT-MSCs transfected with iDSB formed scattered cell aggregates in cell cultures at Day 14. Furthermore, transfected AT-MSCs with iDS and iDSB vectors and treated with BMP4 or RA displayed similar a culture organization compared to the controls with a confluency and fibroblast-like morphology. Scale bars: $500 \mathrm{~mm}$.

AT-MSCs transfected with empty i0 vector and non-transfected AT-MSCs. Tissue samples of AT were isolated from bovine fetuses $(n=9)$ and were pooled separately (each pool from three fetuses). Each pool represented a biological replicate and analyses and experiments were performed in sextuplicate. Pooling tissue samples was performed in an effort to reduce individual biological variation. Furthermore, transfected and control AT-MSCs were treated with $100 \mathrm{ng} / \mathrm{mL}$ of BMP4 or 
$0.1 \mu \mathrm{M}$ of RA for 14 days $(7,8)$. Cells samples at Day 14 were analyzed for the expression of pluripotent genes NANOG and OCT4 and GC genes DAZL, STRA8, BOULE, PIWIL2, cKIT, and FRAGILIS using Q-PCR. Samples were also analyzed for the protein expression of DAZL, STRA8, and NANOG using immunofluorescence.

\section{Isolation and Culture of Bovine Fetal AT-MSCs}

AT-MSCs were isolated following a previously reported protocol that ensured the establishment of MSC cultures that fulfill the minimal criteria for the definition of MSCs $(4,5)$. AT-MSCs were harvested from the omentum of male bovine fetuses $(n=9 ; 7-8$ months of gestation) collected at a local abattoir. Approximately $10 \mathrm{~g}$ of AT was isolated under aseptic conditions and deposited in a phosphate-buffered saline (PBS; Hyclone, Thermo Fisher Scientific, UT, USA) supplemented with $100 \mathrm{IU} / \mathrm{mL}$ of penicillin, $100 \mu \mathrm{g} / \mathrm{mL}$ of streptomycin, and $2.5 \mu \mathrm{g} / \mathrm{mL}$ of amphotericin B (Hyclone). AT samples from three fetuses were pooled and washed twice with PBS and twice with Dulbecco's Modified Eagle Medium (DMEM; Hyclone). Then, AT was digested in $0.5 \%$ collagenase I (Sigma-Aldrich, St. Louis MO, USA) (1 $\mathrm{mL} / \mathrm{g}$ of AT) for $45 \mathrm{~min}$. Collagenase I activity was neutralized with DMEM supplemented with $10 \%$ fetal bovine serum (FBS; Gibco, Waltham, MA, USA), $100 \mathrm{IU} / \mathrm{mL}$ of penicillin, $100 \mu \mathrm{g} / \mathrm{mL}$ of streptomycin, and $2.5 \mu \mathrm{g} / \mathrm{mL}$ of amphotericin B (expansion medium). The disrupted tissue was filtered through $40 \mu \mathrm{m}$ pores and subsequently centrifuged at $400 \mathrm{x}$ g for $5 \mathrm{~min}$. The cell pellet was washed once in DMEM, suspended in an expansion medium, and plated. Following the determination of cell viability, cells were used to initiate the experiments.

\section{Synthesis of Bovine Polycistronic Expression Vectors}

Expression vector pSIN carrying MF2 promoter, cleaving sequences $\mathrm{P} 2 \mathrm{~A}$ and $\mathrm{T} 2 \mathrm{~A}$, puromycin gene, and integrated bovine genes DAZL, STRA8, and BOULE (pSIN-EF2-DAZL-P2ASTRA8-T2A-BOULE-PURO) (iDSB) or DAZL and STRA8 (pSIN-EF2-DAZL-P2A-STRA8-PURO) (iDS), and empty plasmid (pSIN-EF2-P2A-T2A-PURO) (i0) were purchased from GenScript Biotech Corp (Hong Kong, China). Plasmid DNA was extracted from paper by dilution in $300 \mu \mathrm{L}$ of nuclease-free water and placed in a water bath at $50^{\circ} \mathrm{C}$ for $5 \mathrm{~min}$. In order to replicate the plasmid DNA, competent Stbl3 cells (Thermo Fisher Scientific) from a bacterial strain were used. An aliquot of $50 \mu \mathrm{L}$ of the Stbl3 cell-suspension was mixed with 10-100 ng of each DNA plasmid. After several incubation cycles at different temperatures ( $30 \mathrm{~min}$ on ice, $45 \mathrm{~s}$ at $42^{\circ} \mathrm{C}$, and $1 \mathrm{~min}$ on ice), $250 \mu \mathrm{L}$ of warm Super Optimal Broth (SOC) medium (SigmaAldrich) was added and the suspension was incubated for $60 \mathrm{~min}$ at $37^{\circ} \mathrm{C}$ with shaking at $7 \mathrm{~g}$. Subsequently, DNA plasmids were centrifuged for $3 \mathrm{~min}$ at $1,000 \mathrm{~g}$ and $100 \mu \mathrm{L}$ of pellet was removed and seeded with a rake in plates with the Lysogeny broth (LB) medium (Sigma-Aldrich). The plates were incubated for a period of $16 \mathrm{~h}$ at $37^{\circ} \mathrm{C}$ under a humid environment. Colonies obtained after incubation were minced and mixed with the LB medium. A fraction of the suspension was cryopreserved as additional stock using 30\% glycerol. Agar gel electrophoresis was carried out on the diluted fraction to determine the presence of the genes of interest in the bacteria obtained from the colonies in culture by means of the application of restriction enzymes for the detection of DNA fragments. To extract the plasmids, the bacteria was expanded in $100 \mathrm{uL}$ of $\mathrm{LB}$ medium cultures overnight at $37^{\circ} \mathrm{C}$. The purification of the plasmid DNAs was carried out using a NucleoBond Xtra Midi kit (Macherey-Nagel, CA, USA), adding to each pellet $8 \mathrm{~mL}$ of Midiprep RNAse A kit buffer (Thermo Fisher Scientific), and then passing it through a lysis buffer for $5 \mathrm{~min}$ at $25^{\circ} \mathrm{C}$. Subsequently, plasmid filters were equilibrated and washed using the EQU buffer and $5 \mathrm{~mL}$ of elution buffer was applied for DNA decantation. Plasmid DNA precipitation was performed with the addition of $3.5 \mathrm{~mL}$ of isopropanol, which was then removed by adding $2 \mathrm{~mL}$ of $70 \%$ ethanol by centrifugation at $15,000 \mathrm{~g}$ for $5 \mathrm{~min}$ at $4^{\circ} \mathrm{C}$.

\section{AT-MSC Transfection and Treatments With BMP4 and RA}

Transfection of the polycistronic vectors containing combinations of integrated DAZL, STRA8, and BOULE genes was carried out in cultures of bovine fetal AT-MSCs using lipofectamine 2000 (Invitrogen, Carlsbad, CA, USA). AT-MSCs at a concentration of $25 \times 104$ cells $/ \mathrm{mL}$ were seeded in 24 -well plates in an expansion medium until reaching a confluence of $70-90 \%$. After $48 \mathrm{~h}$, the medium was collected and replaced by the Opti-MeM medium supplemented with lipofectamine at a concentration of $1.5 \mathrm{ng} / \mathrm{uL}$. Additionally, $1 \mathrm{ng} / \mathrm{uL}$ of tri-cistronic (iDSB), bi-cistronic (iDS), or empty vector (i0) was added to the medium. Thereafter, the effects of RA and BMP4 on the ATMSCs that were previously transfected and non-transfected were analyzed. AT-MSCs were cultured in a DMEM (low glucose) medium supplemented with $10 \% \mathrm{FBS}, 100 \mathrm{IU} / \mathrm{mL}$ of penicillin, $100 \mu \mathrm{g} / \mathrm{mL}$ of streptomycin, and $0.25 \mu \mathrm{g} / \mathrm{mL}$ of amphotericin B. $\mathrm{RA}$ and BMP4 were supplemented at concentrations of $0.1 \mu \mathrm{M}$ and $100 \mathrm{ng} / \mathrm{mL}$, respectively (7) to each AT-MSC culture for 14 days (Figure 1A). The culture medium containing RA and BMP4 was replaced every 2 days and cells samples at day 14 were analyzed for pluripotency- (NANOG and OCT4) and GC(DAZL, STRA8, BOULE, PIWI, c-KIT, and FRAGILIS) specific marker expression using Q-PCR and for DAZL, STRA8, and NANOG using immunofluorescence.

\section{mRNA Isolation and cDNA Retrotranscription}

Approximately $1 \times 10^{5}$ cells were collected from the transfected and control AT-MSCs and immediately fixed in a lysis buffer (Thermo Fisher Scientific). Total RNA was extracted using the GeneJET RNA purification kit (Thermo Fisher Scientific) according to the manufacturer's instructions. Total RNA was eluted in $50 \mu \mathrm{L}$ of RNAse free water. The concentration and purity of the RNA in each sample was determined using the Qubit RNA assay kit (Life Technologies, Waltham, MA, USA), and genomic DNA was removed using DNase I, RNase-free (Thermo Fisher Scientific). Samples were subjected to reverse transcription using a cDNA synthesis kit (AffinityScript; Agilent Technologies, CA, USA). The reaction protocol consisted of incubation for $5 \mathrm{~min}$ at $25^{\circ} \mathrm{C}, 15 \mathrm{~min}$ at $42^{\circ} \mathrm{C}, 5 \mathrm{~min}$ at $95^{\circ} \mathrm{C}$, and hold at $4^{\circ} \mathrm{C}$ 
TABLE 1 | Sequence of primers used for Q-PCR analysis.

\begin{tabular}{|c|c|c|}
\hline Gene & Nucleotide sequence $\left(5^{\prime}-3^{\prime}\right)$ & $\begin{array}{l}\text { Accession } \\
\text { number }\end{array}$ \\
\hline \multicolumn{3}{|c|}{ Endogenous genes } \\
\hline GAPDH & $\begin{array}{l}\text { Forward CCTTCATTGACCTTCACTACATGG TCTA } \\
\text { Reverse TGGAAGATGGTGATGGCCTITCCATTG }\end{array}$ & NM_001034034.2 \\
\hline$\beta-A C T I N$ & $\begin{array}{l}\text { Forward CGCACCACTGGCATTGTCAT } \\
\text { Reverse TCCAAGGCGACGTAGCAGAG }\end{array}$ & NM_173979.3 \\
\hline \multicolumn{3}{|c|}{ Pluripotency genes } \\
\hline OCT4 & $\begin{array}{l}\text { Forward GAAAGAGAAAGCGGACGAG } \\
\text { Reverse GTGAAAGGAGACCCAGCAG }\end{array}$ & NM_174580.2 \\
\hline NANOG & $\begin{array}{l}\text { Forward TAAGCACAGGGGGCAAAAGT } \\
\text { Reverse ATGGCTAAAAGGGGTGGAGG }\end{array}$ & NM_001025344.1 \\
\hline \multicolumn{3}{|c|}{ Germ cell genes } \\
\hline FRAGILIS & $\begin{array}{l}\text { Forward ATCTGCAGCGAGACCTCTGT } \\
\text { Reverse CCGATGGACATGATGATGAG }\end{array}$ & XM_002697323 \\
\hline DAZL & $\begin{array}{l}\text { Forward TCC AAG TTC ACC AGT TCA GG } \\
\text { Reverse CGT CTG TAT GCT TCT GTC CAC }\end{array}$ & NM_001081725.1 \\
\hline PIWIL2 & $\begin{array}{l}\text { Forward TCGTATTGATGATGTGGATTGG } \\
\text { Reverse GGGAGCAGCAGGATTCAC }\end{array}$ & XM_617223.3 \\
\hline STRA8 & $\begin{array}{l}\text { Forward TGTGCCCAGGTGTTCATCTC } \\
\text { Reverse GGGGACTGTCACCTCATTGG }\end{array}$ & XM_015463130 \\
\hline BOULE & $\begin{array}{l}\text { Forward TGTCACCTGTGCCTITGAATAACC } \\
\text { Reverse TITCAAAAGTGACGAAGCCATACC }\end{array}$ & NM_001102115.1 \\
\hline c-KIT & $\begin{array}{l}\text { Forward TACCAACCAAGGCAGACAA } \\
\text { Reverse CTITGAGGCAAGGAACGC }\end{array}$ & $X M_{-}$ \\
\hline
\end{tabular}

using a TC1000-G gradient thermocycler (SciLogex, Rocky Hill, CT, USA).

\section{Quantitative Polymerase Chain Reaction}

$\beta$-ACTIN and GAPDH (glyceraldehyde 3-phosphate dehydrogenase) were selected as housekeeping genes based on previous analyses from our laboratory that demonstrated high stability in the bfMSC cultures $(4,6)$. Primers were designed using the PrimerExpress software (Applied Biosystems Incorporated, Foster City, CA, USA) (Table 1). Equivalence of amplification efficiencies among all primer-probe sets was confirmed using serial 3-fold dilutions of AT-MSC cDNA. Each PCR reaction $(10 \mu \mathrm{L})$ contained the following: $2 \mathrm{X}$ Brilliant II SYBR Green QPCR master mix (5 $\mu$ L; Agilent Technologies), target forward primer $(200 \mathrm{nM})$, target reverse primer $(200 \mathrm{nM})$, cDNA synthesis reaction $(1 \mu \mathrm{L})$, and nuclease-free PCR-grade water to adjust the final volume. The PCR amplification was carried out in an Eco Real-Time PCR System (Illumina Incorporated, San Diego, CA, USA). Thermal cycling conditions were $95^{\circ} \mathrm{C}$ for $10 \mathrm{~min}$, followed by 40 repetitive cycles at $95^{\circ} \mathrm{C}$ for $30 \mathrm{~s}$, and $60^{\circ} \mathrm{C}$ for $1 \mathrm{~min}$. All reactions were performed in triplicate. In each experiment, the amount of gene expression was recorded as CT values that corresponded to the number of cycles where the fluorescence signal can be detected above a threshold value. The CT averages for each biological replicate were calculated and transformed into relative values through the $\Delta \Delta \mathrm{CT}$ formula (24).

\section{Indirect Immunofluorescence}

The protein expressions of DAZL, STRA8, and NANOG were immunodetected in a bovine fetal (M9) testis obtained at a local abattoir. After extraction from the uterus and placental membranes, the crown-rump length of each fetus was measured in order to estimate the fetal age. Testis samples were collected from bovine fetuses and adults and were fixed in $4 \%$ paraformaldehyde and embedded in paraffin blocks. Testis samples were then sectioned at $5-7 \mathrm{~mm}$ using a microtome, mounted on adhesive coated slides (Newcomer supply; Middleton, Wisconsin) and incubated overnight at $37^{\circ} \mathrm{C}$. Mounted tissues were deparaffinized in xylene and rehydrated in serial alcohol solutions. Slides were subjected to an unmasking protocol by autoclaving at $120^{\circ} \mathrm{C}$ for $30 \mathrm{~min}$ in a citrate buffer $(0.01 \mathrm{M} ; \mathrm{pH}$ 6). Slides were then rinsed two times in PBS and blocked in $2 \%$ bovine serum albumin (BSA) diluted in PBS (pH 7.4) for $1 \mathrm{~h}$ at $4^{\circ} \mathrm{C}$. In order to immunodetect DAZL, STRA8, and NANOG in AT-MSCs, cells were grown in sterile glass coverslips and then fixed using cold methanol at $-20^{\circ} \mathrm{C}$ for $20 \mathrm{~min}$. Subsequently, cells were washed and blocked with $2 \%$ BSA diluted in PBS ( $\mathrm{pH} \mathrm{7.4)}$ for $30 \mathrm{~min}$. The markers were immunodetected using rabbit polyclonal anti-DAZL antibodies (Cat \# ab34139; Abcam, MA, USA), mouse monoclonal antiSTRA8 (Cat\# sc517364; Santa Cruz Biotechnology, CA, USA), or mouse monoclonal anti-NANOG (Cat\# sc-293121; Santa Cruz), diluted in PBS plus 2\% BSA (1:100), and incubated overnight at $4{ }^{\circ} \mathrm{C}$. After three washings with $2 \% \mathrm{BSA}$ in PBS, slides and coverslips were incubated with goat anti-rabbit IgG conjugated with FITC antibodies (Cat\# ab97050; Abcam, USA) or donkey anti-mouse Alexa fluor IgG (Cat\# A21202; Thermo Fisher Scientific) diluted in BSA $2 \%$ for $1 \mathrm{~h}(1: 1,000)$. Finally, slides and coverslips were washed and mounted in DAPI Vectashield mounting media (Cat \# H-1200, CA, USA). The samples were observed and photographed using an epifluorescence microscope and a spectral confocal microscope (Nikon, Japan) with camera connected to a computer.

\section{Data Analysis}

Gene expression data were statistically analyzed using the Infostat software (Cordoba, Argentina). Descriptive column statistics of each data set were performed, including the Shapiro-Wilk normality test. Non-Gaussian distribution of data sets were tested for significant differences using the Kruskal-Wallis test in combination with the Dunn's multiple comparison post-test. The significance level was -0.05 .

\section{RESULTS}

\section{Analysis of the mRNA Levels of Pluripotency and Germ Cell Markers and Immunolocalization of DAZL, STRA8, and NANOG in Bovine Fetal Testis}

Levels of mRNA of pluripotent genes OCT4 and NANOG and GC genes c-KIT, DAZL, STRA8, BOULE, and PIWIL2 were higher $(P<0.05)$ in the adult compared to the fetal 


\section{A}

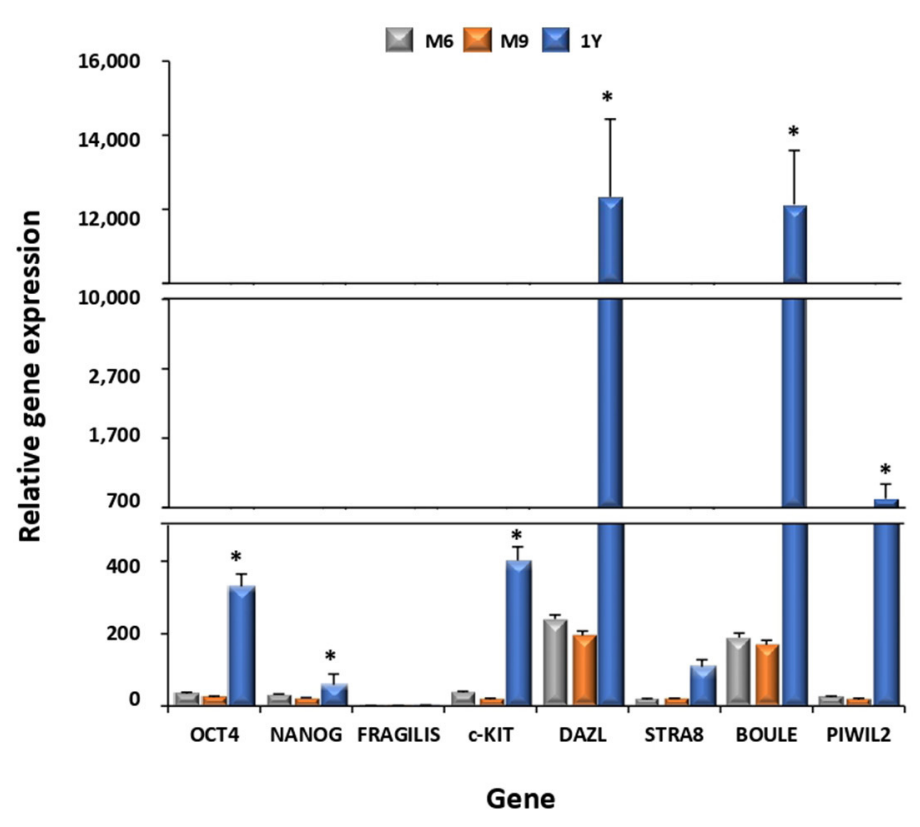

B
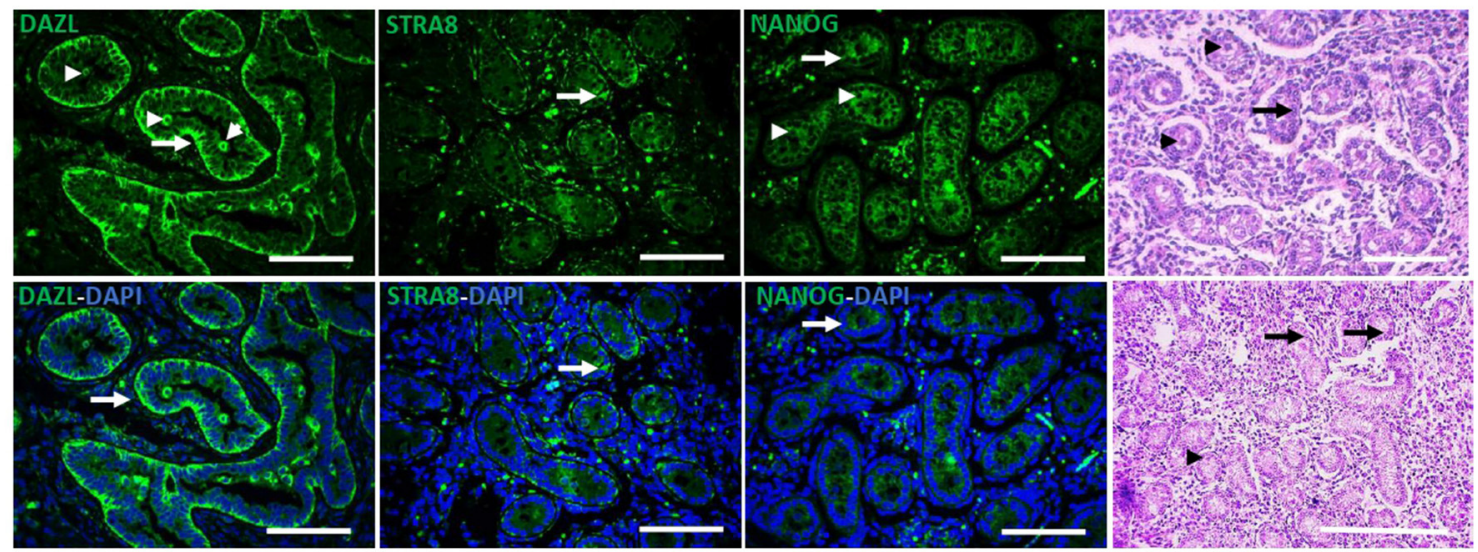

FIGURE 2 | Gene expression analyses of pluripotency and GC markers during bovine testis development and immunodetection of DAZL, STRA8, and BOULE in bovine fetal testis. (A) Levels of mRNA of pluripotent OCT4 and NANOG and GC genes C-KIT, DAZL, STRA8, BOULE, and PIWIL2 were higher (P < 0.05) in adult compared to fetal testis. (B) Sertoli (arrow) and pre-spermatogonial (arrowhead) cells are indicated in epifluorescence and H\&E staining of bovine fetal testis (M9). An intense immunosignal associated to DAZL located in Sertoli and pre-spermatogonial cells was detected in the fetal testis. STRA8 immunosignal was observed in the spotted areas mainly in the basal compartment of the seminiferous tubules. NANOG immunosignal was observed across the spermatogenic epithelium with high intensity in Sertoli and pre-spermatogonial cells. $\left(^{\star}\right)$ indicates significant differences $(P<0.05)$ for gene expression levels between the developmental stages for each gene. Scale bars: $200 \mu \mathrm{m}$.

testis (Figure 2A). No significant differences in the mRNA levels of these genes were detected in testis between fetal stages. The expression of DAZL, STRA8, and NANOG was thereafter evaluated during fetal testicular tissue in order to determine its expression in association with gonocytes, pre-spermatogonia, and sertoli cells, which represent the initial stage of germ cell differentiation and a stage more similar to MSC differentiation. An intense immunosignal associated to DAZL probably located in pre-spermatogonial cells was detected in the peripheral areas of seminiferous tubules (Figure 2B) in a 9-month fetal testis. STRA8 immunosignal was observed in the spotted areas mainly in the basal compartment of the seminiferous tubules. Furthermore, we immunodetected NANOG across the spermatogenic epithelium with a high intensity in the Sertoli and pre-spermatogonial cells. 


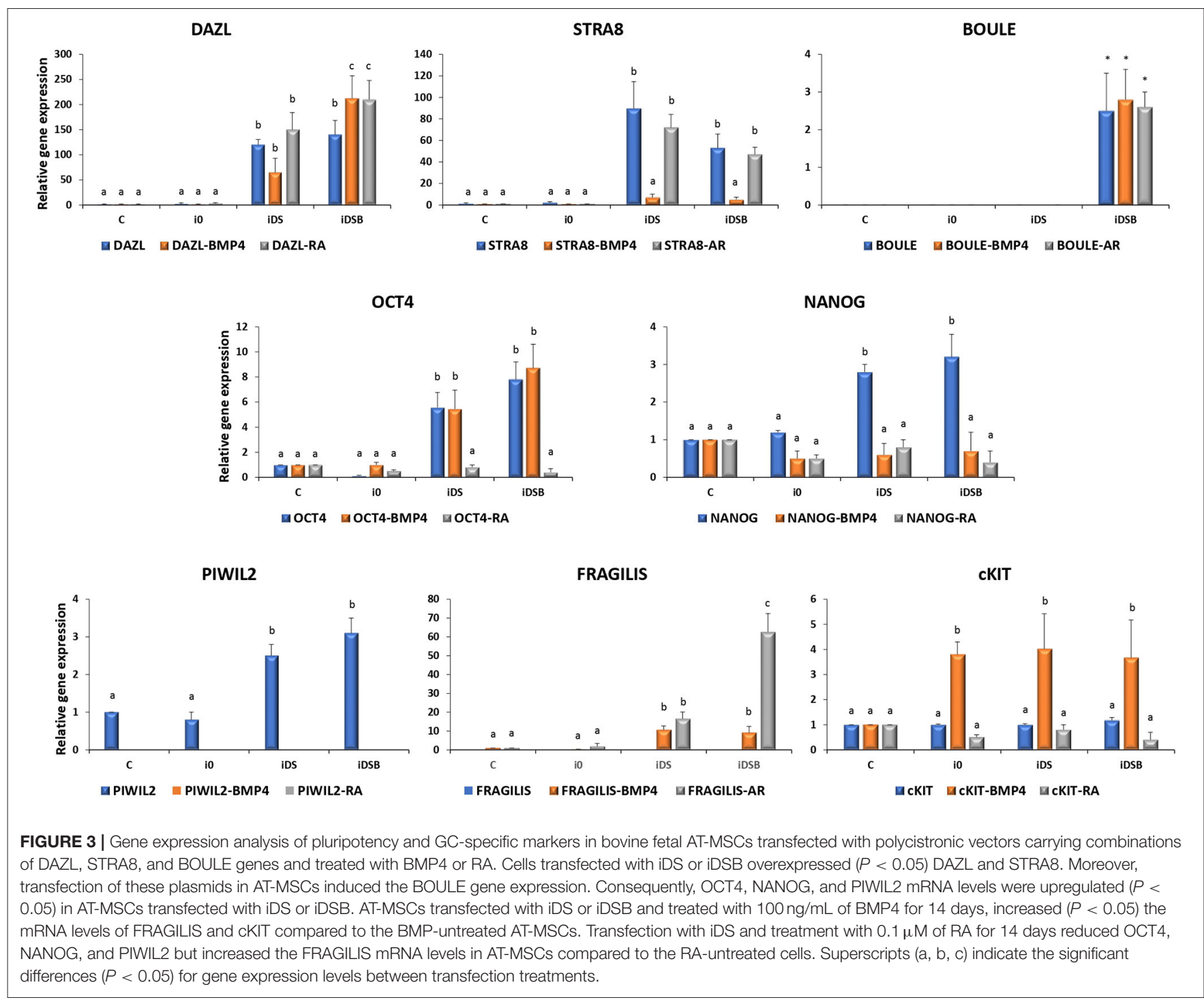

\section{Morphological Characterization of Bovine Fetal Mesenchymal Stem Cells Transfected With Polycistronic Vectors Carrying Combinations of DAZL, STRA8, and BOULE Genes and Treated With Bone Morphogenetic Factor 4 or Retinoic Acid}

Transfected AT-MSCs had a similar fibroblast-like morphology and culture organization compared to the control ATMSCs (Figure 1B). AT-MSCs transfected with tri-cistronic iDSB formed scattered cell aggregates in cell cultures. Furthermore, the transfected AT-MSCs with bi-cistronic and tri-cistronic vectors and treated with BMP4 or RA displayed a similar culture organization compared to the controls with confluency and fibroblast-like morphology at Day 14.
Analysis of the mRNA Levels and Immunolocalization of Pluripotency and Germ Cell-Specific Markers in Bovine Fetal Mesenchymal Stem Cells Transfected With Polycistronic Vectors Carrying Combinations of DAZL, STRA8, and BOULE Genes and Treated With Bone Morphogenetic Factor $\mathbf{4}$ or Retinoic Acid

Cells transfected with iDS or iDSB overexpressed $(\mathrm{P}<0.05)$ DAZL (120.3- and 140.1-fold, respectively) and STRA8 (89.6and 53.1-fold, respectively) Figure 3. Moreover, transfection of iDSB plasmid in AT-MSCs induced the BOULE gene expression (1-fold). Consequently, the OCT4 and NANOG mRNA levels were upregulated $(P<0.05)$ in the AT-MSCs transfected with 


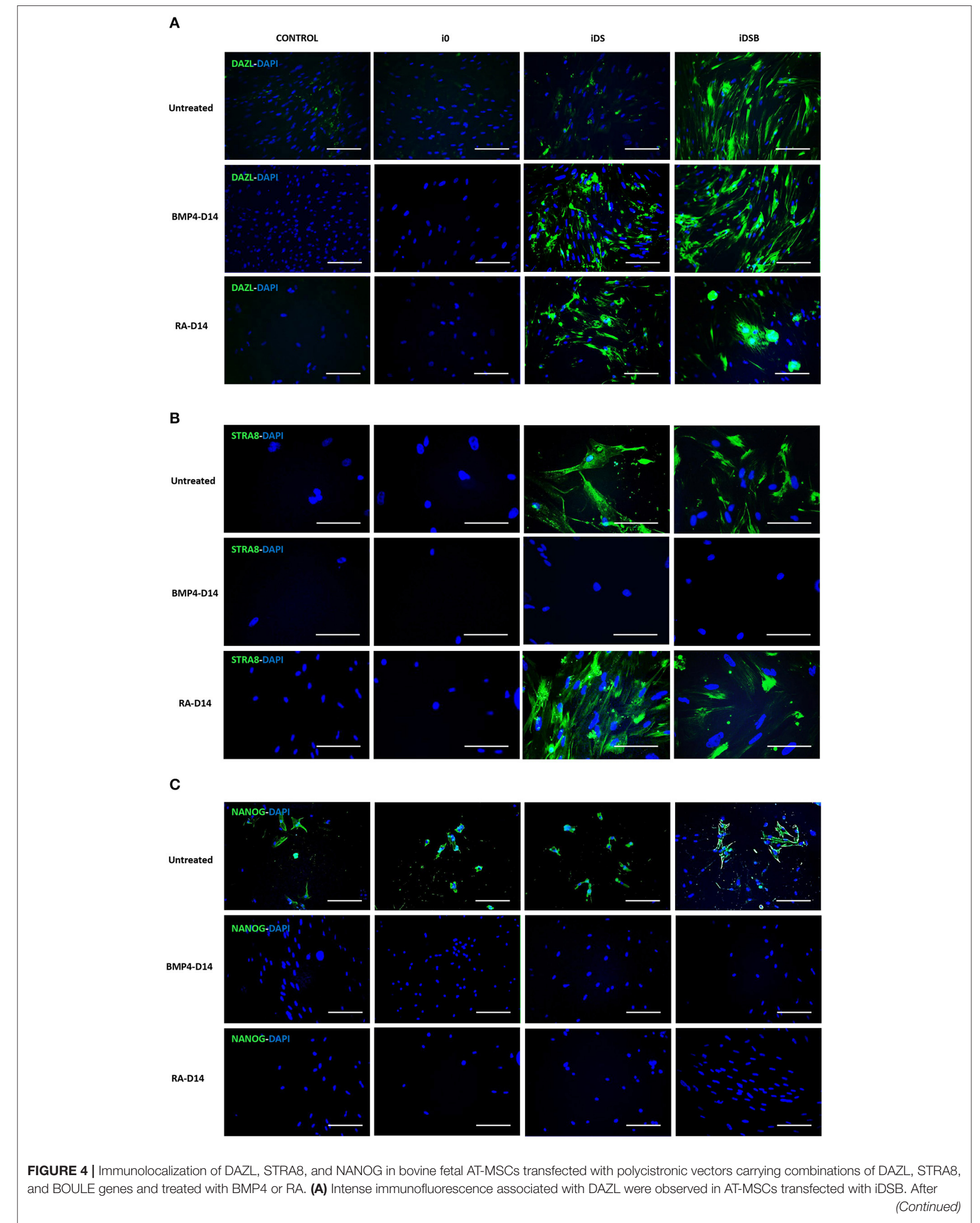


FIGURE 4 | treatment with BMP4 or RA for 14 days, immunoreactivity associated to DAZL remained in AT-MSCs transfected with iDSB. (B) STRA8 was immunolocalized in AT-MSCs transfected with iDS and iDSB and after treatment with RA. (C) Immunosignal associated with NANOG was observed in control cells and transfected AT-MSCs, however, BMP4 and RA treatments suppressed the NANOG immunosignal after 14 days of culture. Scale bars: $100 \mu \mathrm{m}$.

iDS (5.6- and 2.8-fold, respectively) or iDSB (7.8- and 3.2-fold, respectively). Moreover, these cells increased $(P<0.05)$ the PIWIL2 mRNA levels when transfected with iDS or iDSB (2.5and 3.1-fold, respectively).

AT-MSCs, transfected with iDS and treated with $100 \mathrm{ng} / \mathrm{mL}$ of BMP4 for 14 days, had similar $(P<0.05)$ mRNA levels of DAZL (65.1-fold), BOULE (1.3-fold), and OCT4 (5.5-fold) compared to the BMP4-untreated cells. However, this treatment increased $(P<0.05)$ the mRNA levels of FRAGILIS $(10.6$-fold) and cKIT (4-fold) and reduced $(P<0.05)$ the expression of STRA8 (7.1fold), NANOG (0.6-fold), and PIWIL2 (undetected) in ATMSCs compared to the BMP4-untreated cells. In comparison, AT-MSCs, transfected with iDSB and treated with BMP4 for 14 days, increased $(P<0.05)$ the expression of DAZL $(212.3$ fold), FRAGILIS (9.1-fold), and cKIT (3.7-fold) compared to the BMP4-untreated controls. Moreover, cells transfected with iDSB and treated with BMP4 had similar $(P<0.05)$ mRNA levels of BOULE (2.8-fold) and OCT4 (8.7-fold) but lower $(P<0.05)$ mRNA levels of STRA8 (5.2-fold), NANOG (0.7-fold), and PIWIL2 (undetected) compared to the BMPuntreated AT-MSCs.

Transfection with iDS and treatment with $0.1 \mu \mathrm{M}$ of RA for 14 days resulted in similar mRNA levels of DAZL (150.1fold), STRA8 (72.1-fold), BOULE (1.2-fold), and cKIT (0.8-fold) compared to the RA-untreated cells. Moreover, this treatment reduced OCT4 (0.8-fold), NANOG (0.8-fold), and PIWIL2 (undetected) but increased the FRAGILIS (16.6-fold) mRNA levels in AT-MSCs compared to the RA-untreated cells. AT-MSCs transfected with iDSB and exposed to RA for 14 days increased $(P<0.05)$ DAZL (210-fold) and FRAGILIS (62.7-fold) and had similar $(P>0.05)$ levels of STRA8 (47.5-fold), BOULE (2.6-fold), and cKIT (0.4-fold) mRNA levels compared to the RA-untreated cells. However, this treatment reduced $(P<0.05)$ the OCT4 (0.4-fold), NANOG (0.4-fold), and PIWIL2 (undetected) mRNA levels in AT-MSCs compared to the RA-untreated controls.

Intense immunofluorescence associated with DAZL, STRA8, and NANOG were observed in the AT-MSCs transfected with iDSB (Figures 4A,C). A weak DAZL immunosignal was also observed in the AT-MSCs transfected with iDS. In addition, STRA8 and NANOG were immunolocalized in the AT-MSCs transfected with iDS (Figures 4B,C). Immunosignal associated with NANOG was also observed in the control cells and ATMSCs transfected with i0 (Figure 4C). After treatment with BMP4 for 14 days, immunoreactivity associated to DAZL remained in the AT-MSCs transfected with iDSB; however, this treatment resulted in the lack of STRA8 and NANOG immunosignal. Similarly, AT-MSCs transfected with iDS and treated with BMP4 were not immunoreactive to STRA8 and NANOG. Furthermore, after treatment with RA for 14 days, immunoreactivity to DAZL and STRA8 was observed in the AT-MSCs transfected either with iDSB or iDS. However, treatment with RA suppressed the NANOG immunoreactivity in these cells.

\section{DISCUSSION}

The gene expression patterns and tissue-specific localization of selected GC markers were analyzed in the bovine testis during the late fetal and adult stages. We found that the gene expression levels of OCT4, NANOG, c-KIT, DAZL, STRA8, BOULE, and PIWIL2 increased remarkably in the bovine testis from the fetal to adult stage. It has been reported that the OCT4 and NANOG expression expands from gonocytes in the neonatal testis to spermatocytes and round spermatids after sexual maturation, which may explain the increased expression levels found in the adult stage $(25,26)$. In concordance with these results, we immunodetected NANOG across the spermatogenic epithelium of the fetal testis with a high intensity in the Sertoli and pre-spermatogonial cells. Even though the roles of FRAGILIS and c-KIT have not been deeply studied in cattle, the expression of both genes occurs early in development, where they activate important mediators of postnatal meiotic and post-meiotic GC (26). In addition to the increasing expression levels of DAZL in the bovine testis during development, our analyses immunodetected DAZL mainly in the peripheral areas of bovine fetal seminiferous tubules associated to Sertoli and pre-spermatogonial cells. Similarly, DAZL gene expression levels have been shown to increase from pubertal to sexually mature sheep and dog testis, where it has been located in spermatogonia, primary and secondary spermatocytes (22, 27, 28). Moreover, we observed an immune signal associated to STRA8 in the spotted areas across the germinal epithelium in seminiferous tubules, showing a higher intensity in the basal compartment. STRA8 expression levels have been reported to peak at 6 months of age in goat testis and has been associated to the onset of meiosis with the highest intensity levels in preleptotene/early leptotene spermatocytes $(29,30)$. Levels of the BOULE expression in goat has been reported to increase in the adult compared to the immature testis, which may be associated to an increasing number of BOULE positive spermatogonial cells at the adult stage (31). Furthermore, the PIWIL2 gene expression pattern was in concordance to what was previously described in ovine, where high expression levels have been detected in sexually mature gonadal tissues, mainly localized in spermatogonia, primary spermatocyte, and oocyte (32). Thus, the overall increased expression levels of DAZL, STRA8, and BOULE in the adult testis and cell-specific distribution of DAZL and STRA8 in the fetal testis suggest that these genes are important components of the regulatory network that control the in vivo differentiation of bovine GCs.

In order to mimic the gene-regulatory complex that activates the GC development in vivo, we then sought to develop a 
multigene overexpression system using polycistronic vectors carrying combinations of DAZL, STRA8, and BOULE to induce GC differentiation of bovine fetal AT-MSCs. Polycistronic constructs have been successfully used for the co-expression of genes in a wide variety of biotechnological applications including iPSC production and gene therapy (33-35). In the current study, transcription of polycistronic vectors in bovine fetal AT-MSCs induced a positional expression effect in second position gene STRA8 in tri-cistronic vectors. Tri-cistronic constructs induce a higher expression in first positions genes compared to second and third position sequences, which may explain why it was lower in the STRA8 expression. Moreover, a gradual decrease in the transcribed mRNA along the vector due to the naturally occurring ribosome drop-off has been described in long sequence transcripts (36). Despite the increased transcript length, BOULE gene located in the third position in tri-cistrionic vector increased its expression compared to the controls.

Nevertheless, in the present study, overexpression of DAZL and STRA8 in bi-cistronic and DAZL, STRA8, and BOULE in tricistronic vectors resulted in the upregulation of OCT4, NANOG, and PIWIL2 in bovine fetal AT-MSCs. During spermatogenesis, DAZL expression overlaps with OCT4 and NANOG, until OCT4 is downregulated when cells enter meiosis at the type B spermatogonia stage and NANOG levels decrease when migrating PGCs reach the gonad (37). Previously, it has been reported that a combined or individual overexpression of DAZL, STRA8, or BOULE in goat BM-MSCs resulted in the upregulation of OCT4 $(22,23)$. Even though the molecular mechanism of STRA8 remains unclear, recently, it has been reported that STRA8 may regulate spermatogenesis via the Cdl4Clu4A-Ddb1 ubiquitinated degradation axis in a proliferating cell nuclear antigen (PCNA)-dependent manner (38). On the other hand, DAZL has been associated to several molecules including the polyribosomes and poly(A)-binding proteins (PABPs), which suggests its participation in several aspects of protein synthesis including mRNA stability, transportation, localization, or translation (39). Despite the reported concomitant activities of DAZL and BOULE in spermatogenesis (21), our data showed no synergistic effect after BOULE inclusion in tricistronic vectors. Although the mechanism whereby DAZL or STRA8 regulates the level of OCT4 and NANOG is currently unclear, our data suggest that STRA8 and DAZL may regulate pluripotency through the activation of these transcription factors.

Treatment with BMP4 increased the expression of DAZL in bovine fetal AT-MSCs transfected with tri-cistrionic vector. We have previously reported that the un-transfected bovine fetal AT-MSCs treated with the same concentration of BMP4 increased the DAZL mRNA levels at Day 14 of culture (7). BMP4 activity is mediated by receptor ALK3 and transducer SMAD5, exerting both mitogenic and differentiative effects (40). In the present study, while BMP4 repressed the NANOG expression, this treatment also activated FRAGILIS and increased the cKIT gene expression levels in bovine fetal AT-MSCs. BMP4 control over the FRAGILIS expression has been widely studied in mice development, mediated through BMP4 signaling secreted from the extra-embryonic ectoderm (12). Evidence sustaining the
BMP4-activation of FRAGILIS is supported by studies showing a lack of the FRAGILIS positive cells in BMP4-null embryos and significant reduction in BMP4 heterozygous embryos (12). Moreover, c-KIT is also be activated by BMP4 in spermatogonial cells, via Alk3 and the R-Smad Smad5 stimulation, resulting in both mitogenic and differentiative effects (40). Overall, these data suggest that BMP4 effect may control differentiation of bovine fetal AT-MSCs by reducing pluripotency and activating gene expression of early GC markers including DAZL, FRAGILIS, and c-KIT.

Treatment with RA for 14 days increased the expression of DAZL and FRAGILIS, and maintained the mRNA levels of STRA8 in bovine fetal AT-MSCs transfected with bi-cistronic and tri-cistronic vectors. Moreover, RA treatment repressed the expression of OCT4 and NANOG in these cells. Our previous analyses in bovine fetal BM-MSC showed that the NANOG levels were not affected by RA; however, this treatment increased the expression of DAZL and reduced the expression of OCT4 (7). $\mathrm{RA}$ is a multipurpose factor that controls the meiotic entrance of PGCs in the genital cords by controlling the Cyp26b1 enzyme expression (15). RA activity has also been reported in the ESCs resulting in the increased gene expression levels of several GC genes including FRAGILIS and STRA8 (41). Activity of RA is exerted inside the nucleus during gametogenesis through their binding to the RA receptors (RAR) in several cell types including Sertoli cells $(\mathrm{RAR} \alpha)$, round spermatids $(\mathrm{RAR} \beta)$, and type A spermatogonia (RAR $\gamma$ ) (42). Even though the signaling pathway of RA has not been entirely described in MSCs, these results suggest that RA partially activates the GC gene expression pattern by upregulating DAZL and STRA8 in overexpressing ATMSCs. Recently, it has been reported that the BMP4 and RA signaling pathways play opposing roles in GC formation (43). BMP4 regulates PGC formation through histone acetylation and DNA methylation in the DAZL gene. In comparison, RA binds RAR $\alpha$ which is regulated by STRA8 promoter activity during SSC formation (43).

In conclusion, gene expression and immunohistochemical data suggest that DAZL, STRA8, and BOULE are important components of the regulatory network that control the in vivo differentiation of bovine GCs. Overexpression of DAZL, STRA8, and BOULE induced the upregulation of pluripotency markers and PIWIL2 in the transfected bovine fetal AT-MSCs. BMP4 and RA partial activation of the GC gene expression suggests that both factors possess common targets but induce different gene expression effects during the GC differentiation in overexpressing bovine fetal AT-MSCs.

\section{DATA AVAILABILITY STATEMENT}

The raw data supporting the conclusions of this article will be made available by the authors, without undue reservation.

\section{ETHICAL STATEMENT}

The animal study was reviewed and approved by Bioethical Committee of the National Commission 
for Scientific and Technology Research from Chile (Fondecyt).

\section{AUTHOR CONTRIBUTIONS}

MDR, CGT, MV-G, VHP, and OAP contributed to the conception and design of the study, acquisition of data, analysis, and interpretation of data, drafting the article, writing, review and editing, and revising the article critically for important intellectual content. PC, AG-M,

\section{REFERENCES}

1. West FD, Shirazi R, Mardanpour P, Ozcan S, Dinc G, Hodges DH, et al. In vitro-derived gametes from stem cells. Semin Reprod Med. (2013) 31:33-8. doi: 10.1055/s-0032-1331795

2. Volarevic V, Bojic S, Nurkovic J, Volarevic A, Ljujic B, Arsenijevic N, et al. Stem cells as new agents for the treatment of infertility: current and future perspectives and challenges. Biomed Res Int. (2014) 2014:507234. doi: $10.1155 / 2014 / 507234$

3. Hill JR, Dobrinsky I. Male germ cell transplantation in livestock. Reprod Fertil Dev. (2006) 18:13-8. doi: 10.1071/RD05123

4. Cortes Y, Ojeda M, Araya D, Dueñas F, Fernández MS, Peralta OA. Isolation and multilineage differentiation of bone marrow mesenchymal stem cells from abattoir-derived bovine fetuses. BMC Vet Res. (2013) 9:133. doi: 10.1186/1746-6148-9-133

5. Díaz P, Cuevas F, Peralta OA. GFP labelling and epigenetic enzyme expression of bone marrow-derived mesenchymal stem cells from bovine foetuses. Res Vet Sci. (2015) 99:120-8. doi: 10.1016/j.rvsc.2014.12.019

6. Dueñas F, Becerra V, Cortes Y, Vidal S, Sáenz L, Palomino J, et al. Hepatogenic and neurogenic differentiation of bone marrow mesenchymal stem cells from abattoir-derived bovine fetuses. BMC Vet Res. (2014) 10:154. doi: 10.1186/1746-6148-10-154

7. Cortez J, Bahamonde J, De Los Reyes M, Palomino J, Torres CG, Peralta OA. In vitro differentiation of bovine bone marrow-derived mesenchymal stem cells into male germ cells by exposure to exogenous bioactive factors. Reprod Domest Anim. (2018) 53:700-9. doi: 10.1111/rda.13160

8. Segunda MN, Bahamonde J, Muñoz I, Sepulveda S, Cortez J, De Los Reyes M, et al. Sertoli cell-mediated differentiation of bovine fetal mesenchymal stem cells into germ cell lineage using an in vitro co-culture system. Theriogenology. (2019) 130:8-18. doi: 10.1016/j.theriogenology.2019.02.034

9. Huaman O, Bahamonde J, Cahuascanco B, Jervis M, Palomino J, Torres CG, et al. Immunomodulatory and immunogenic properties of mesenchymal stem cells derived from bovine fetal bone marrow and adipose tissue. Res Vet Sci. (2019) 124:212-22. doi: 10.1016/j.rvsc.2019.03.017

10. Zhang ZY, Teoh SH, Hui JHP, Fisk NM, Choolani M, Chan JKY. The potential of human fetal mesenchymal stem cells for off-the-shelf bone tissue engineering application. Biomaterials. (2012) 33:2656-72. doi: 10.1016/j.biomaterials.2011.12.025

11. Lawson KA, Hage WJ. Clonal analysis of the origin of primordial germ cells in the mouse. Ciba Found Symp. (1994) 182:68-84. doi: 10.1002/9780470514573.ch5

12. Saitou M, Barton SC, Surani MA. A molecular programme for the specification of germ cell fate in mice. Nature. (2002) 418:293-0. doi: $10.1038 /$ nature00927

13. Hua J, Sidhu K. Recent advances in the derivation of germ cells from the embryonic stem cells. Stem Cells Dev. (2008) 3:399-11. doi: 10.1089/scd.2007.0225

14. Yen PH. Putative biological functions of the DAZL family. Int J Androl. (2004) 27:125-9. doi: 10.1111/j.1365-2605.2004.00469.x

15. Koubova J, Menke DB, Zhou Q, Capel B, Griswold MD, Page DC. Retinoic acid regulates sex-specific timing of meiotic initiation in mice. Proc Natl Acad Sci USA. (2006) 103:2474-9. doi: 10.1073/pnas.0510813103 and JC performed the experiments and analyzed the data. All authors contributed to the article and approved the submitted version.

\section{FUNDING}

This study was supported by grants 1191114 and 1161251 from the National Commission for Scientific and Technology Research (Fondecyt) from the Ministry of Education, Government of Chile.

16. Anderson EL, Baltus AE, Roepers-Gajadien HL, Hassold TJ, de Rooij DG, van Pelt AM, et al. Stra8 and its inducer, retinoic acid, regulate meiotic initiation in both spermatogenesis and oogenesis in mice. Proc Natl Acad Sci USA. (2008) 105:14976-80. doi: 10.1073/pnas.0807297105

17. Baltus AE, Menke DB, Hu Y-C, Goodheart ML, Carpenter AE, de Rooij $\mathrm{DG}$, et al. In germ cells of mouse embryonic ovaries, the decision to enter meiosis precedes premeiotic DNA replication. Nat Genet. (2006) 38:1430-4. doi: $10.1038 / \mathrm{ng} 1919$

18. Riggiu M, Speed R, Taggart M, McKay SJ, Kilanowski F, Saunders P, et al. The mouse Dazl gene encodes a cytoplasmic protein essential for gametogenesis. Nature. (1997) 398:73-7. doi: 10.1038/37987

19. Lin Y, Page DC. Dazl deficiency leads to embryonic arrest of germ cell development in XY C57BL/6 mice. Dev Biol. (2005) 288:309-16. doi: 10.1016/j.ydbio.2005.06.032

20. VanGompel MJ, Xu EY. A novel requirement in mammalian spermatid differentiation for the DAZ-family protein Boule. Hum Mol Genet. (2010) 19:2360-9. doi: 10.1093/hmg/ddq109

21. Fu XF, Cheng SF, Wang LQ, Yin S, De Felici M, Shen W. DAZ family proteins, key players for germ cell development. Int J Biol Sci. (2015) 11:1226-35. doi: 10.7150/ijbs.11536

22. PZ Li, Yan GY, Han L, Pang J, Zhong BS, Zhang GM, et al. Overexpression of STRA8, BOULE, and DAZL genes promotes goat bone marrow-derived mesenchymal stem cells in vitro transdifferentiation toward putative male germ cells. Reprod Sci. (2017) 24:300-12. doi: 10.1177/1933719116654990

23. Zhang YL, Li PZ, Pang J, Wan YJ, Zhang GM, Fan YX, et al. Induction of goat bone marrow mesenchymal stem cells into putative male germ cells using mRNA for STRA8, BOULE and DAZL. Cytotechnology. (2019) 71:563-72. doi: 10.1007/s10616-019-00304-7

24. Vandosempele J, De Preter K, Pattyn F, Poppe B, Van Roy N, De Paepe A, et al. Accurate normalization of real-time quantitative RT-PCR data by geometric averaging of multiple internal control genes. Genome Biol. (2002) 3:1-12. doi: 10.1186/gb-2002-3-7-research0034

25. Fujihara M, Kim SM, Minami N, Yamada M, Imai H. Characterization and in vitro culture of male germ cells from developing bovine testis. J Reprod Dev. (2011) 57:355-64. doi: 10.1262/jrd.10-185M

26. De Sousa AF, Godoy NC, Dos Santos D. Step by step about germ cells development in canine. Animals. (2021) 11:598. doi: 10.3390/ani11030598

27. Yuan Z, Luo J, Wang L, Li F, Li W, Yue X. Expression of DAZL Gene in selected tissues and association of its polymorphisms with testicular size in Hu sheep. Animals. (2020) 10:740. doi: 10.3390/ani10040740

28. Pieri NCG, Souza AF, Mancanares ACF, Roballo KCS, Casals JB, Ambrosio $\mathrm{CE}$, et al. Immunolocalization of proteins in the spermatogenesis process of canine. Reprod Dom Anim. (2017) 52:170-6. doi: 10.1111/rda.12848

29. Zhou Q, Nie R, Li Y, Friel P, Mitchell D, Hess RA, et al. Expression of stimulated by retinoic acid gene 8 (Stra8) in spermatogenic cells induced by retinoic acid: an in vivo study in vitamin A-sufficient postnatal murine testes. Biol Reprod. (2008) 79:35-42. doi: 10.1095/biolreprod.107. 066795

30. Faucette AN, Maher VA, Gutierrez MA, Jucker JM, Yates DC, Welsh $\mathrm{TH}$, et al. Temporal changes in histomorphology and gene expression in goat testes during postnatal development. J Anim Sci. (2014) 92:4440-8. doi: $10.2527 /$ jas.2014-7903 
31. Li M, Liu C, Zhu H, Sun J, Yu M, Niu Z, et al. Expression pattern of Boule in dairy goat testis and its function in promoting the meiosis in male germline stem cells (mGSCs). J Cell Biochem. (2013) 114:294-02. doi: 10.1002/jcb. 24368

32. Li C, Liu Q, Wang X, Hu W, Han D, Mwashigadi Mwacharo J, et al. Expression and localization of PIWI proteins in testis and ovary of domestic sheep. Czech J Anim. (2020) 65:86-96. doi: 10.17221/7/2020-CJAS

33. Szymczak AL, Workman CJ, Wang Y, Vignali KM, Dilioglou S, Vanin $\mathrm{EF}$, et al. Correction of multi-gene deficiency in vivo using a single 'selfcleaving' 2A peptide-based retroviral vector. Nat Biotechnol. (2004) 22:589-94. doi: $10.1038 /$ nbt957

34. Takahashi K, Yamanaka S. Induction of pluripotent stem cells from adult human fibroblasts by defined factors. Cell. (2007) 131:861-72. doi: 10.1016/j.cell.2007.11.019

35. Vaseghi HR, Yin C, Zhou Y, Jiandong L, Qian L. Generation of an inducible fibroblast cell line for studying direct cardiac reprogramming. Genesis. (2016) 54:398-06, doi: 10.1002/dvg.22947

36. Liu Z, Chen O, Blake Joseph Wall J, Zheng M, Zhou Y, Wang L, et al. Systematic comparison of $2 \mathrm{~A}$ peptides for cloning multi-genes in a polycistronic vector. Sci Rep. (2017) 7:2193. doi: 10.1038/s41598-017-02460-2

37. Saitou M, Miyauchi H. Gametogenesis from pluripotent stem cells. Cell Stem Cell. (2016) 18:721-35. doi: 10.1016/j.stem.2016.05.001

38. Niu C, Guo J, Shen X, Ma S, Xia M, Xia J, et al. Meiotic gatekeeper STRA8 regulates cell cycle by interacting with SETD8 during spermatogenesis. J Cell Mol Med. (2020) 24:4194-211. doi: 10.1111/jcmm. 15080

39. Tsui S, Dai T, Warren ST, Salido EC, Yen PH. Association of the mouse infertility factor DAZL1 with actively translating polyribosomes. Biol Reprod. (2000) 62:1655-60. doi: 10.1095/biolreprod62.6.1655
40. Pellegrini M, Grimaldi P, Rossi P, Geremia R, Dolci S. Developmental expression of BMP4/ALK3/SMAD5 signaling pathway in the mouse testis: a potential role of BMP4 in spermatogonia differentiation. J Cell Sci. (2003) 116:3363-72. doi: 10.1242/jcs.00650

41. Eskandari N, Moghaddam MH, Atlasi MA, Mahabadi JA, Taherian A, Nikzad $H$. The combination of retinoic acid and estrogen can increase germ cells genes expression in mouse embryonic stem cells derived primordial germ cells. Biologicals. (2018) 56:39-44. doi: 10.1016/j.biologicals.2018. 10.001

42. Vernet N, Dennefeld C, Rochette-Egly C, Oulad-Abdelghani M, Chambon P, Ghyselinck NB, et al. Retinoic acid metabolism and signaling pathways in the adult and developing mouse testis. Endocrinology. (2006) 147:96-110. doi: 10.1210/en.2005-0953

43. Zuo Q, Jin J, Jin K, Sun C, Song J, Zhang Y, et al. Distinct roles of retinoic acid and BMP4 pathways in the formation of chicken primordial germ cells and spermatogonial stem cells. Food Funct. (2019) 10:7152. doi: 10.1039/C9FO01 $485 \mathrm{C}$

Conflict of Interest: The authors declare that the research was conducted in the absence of any commercial or financial relationships that could be construed as a potential conflict of interest.

Copyright (C 2021 Cordero, Guerrero-Moncayo, De los Reyes, Varas-Godoy, Cortez, Torres, Parraguez and Peralta. This is an open-access article distributed under the terms of the Creative Commons Attribution License (CC BY). The use, distribution or reproduction in other forums is permitted, provided the original author(s) and the copyright owner(s) are credited and that the original publication in this journal is cited, in accordance with accepted academic practice. No use, distribution or reproduction is permitted which does not comply with these terms. 